\title{
Weight Status in Persons with Multiple Sclerosis: Implications for Mobility Outcomes
}

\author{
Lara A. Pilutti, ${ }^{1}$ Deirdre Dlugonski, ${ }^{1}$ John H. Pula, ${ }^{2}$ and Robert W. Motl1 \\ ${ }^{1}$ Department of Kinesiology and Community Health, University of Illinois at Urbana-Champaign, 233 Freer Hall, Urbana, \\ IL 61801, USA \\ ${ }^{2}$ School of Medicine, University of Illinois at Peoria, Peoria, IL 61603, USA
}

Correspondence should be addressed to Robert W. Motl, robmotl@illinois.edu

Received 14 July 2012; Accepted 22 August 2012

Academic Editor: Jordi Salas-Salvadó

Copyright (C) 2012 Lara A. Pilutti et al. This is an open access article distributed under the Creative Commons Attribution License, which permits unrestricted use, distribution, and reproduction in any medium, provided the original work is properly cited.

\begin{abstract}
The accumulation of excess body weight may have important health and disease consequences for persons with multiple sclerosis (MS). This study examined the effect of weight status on mobility using a comprehensive set of mobility outcomes including ambulatory performance (timed 25-foot walk, T25FW; 6-minute walk, 6MW; oxygen cost of walking, $C_{\mathrm{w}}$; spatiotemporal parameters of gait; self-reported walking impairment, Multiple Sclerosis Walking Scale-12 (MSWS-12); and free-living activity, accelerometry) in 168 ambulatory persons with MS. Mean (SD) BMI was $27.7(5.1) \mathrm{kg} / \mathrm{m}^{2}$. Of the 168 participants, $31.0 \%$ were classified as normal weight $\left(\mathrm{BMI}=18.5-24.9 \mathrm{~kg} / \mathrm{m}^{2}\right), 36.3 \%$ were classified as overweight $\left(\mathrm{BMI}=25.0-29.9 \mathrm{~kg} / \mathrm{m}^{2}\right)$, and $32.7 \%$ were classified as obese, classes I and II $\left(\mathrm{BMI}=30-39.9 \mathrm{~kg} / \mathrm{m}^{2}\right)$. There were no significant differences among BMI groups on T25FW and $6 \mathrm{MW}, C_{\mathrm{w}}$, spatiotemporal gait parameters, MSWS-12, or daily step and movement counts. The prevalence of overweight and obesity in this sample was almost 70\%, but there was not a consistent nor significant impact of BMI on outcomes of mobility. The lack of an effect of weight status on mobility emphasizes the need to focus on and identify other factors which may be important targets of ambulatory performance in persons with MS.
\end{abstract}

\section{Introduction}

Currently, the combined prevalence of overweight (BMI, body mass index $\left.\geq 25 \mathrm{~kg} / \mathrm{m}^{2}\right)$ and obesity $\left(\mathrm{BMI} \geq 30 \mathrm{~kg} / \mathrm{m}^{2}\right)$, defined by the World Health Organization (WHO) [1,2], has ranged from approximately 50 to 65 percent in large samples of persons with MS [3-5]. These estimates may not differ considerably from those of nondiseased adult populations [6], but weight status may have important consequences for mobility and the accumulation of disability in persons with MS [4].

Mobility, assessed by a variety of measures, is compromised in nondiseased persons who carry excess body weight. Compared to normal weight persons, those who were obese walked at slower speeds and covered less distance during a 6-minute walk (6MW) test [7]. A higher oxygen cost of walking $\left(C_{\mathrm{w}}\right)$ has been established in nondiseased obese adults compared to adults of normal weight [8]. Altered gait kinematics including slower walking speed, shorter stride length, and more time spent in stance phase and double support during walking have further been observed in obese compared to normal-weight adults [9]. Such results in nondiseased populations would support the idea that weight status might similarly be a source of mobility dysfunction seen in persons with MS [10-13].

Our group has recently investigated the prospective relationship between self-reported BMI and disability, assessed by the Patient Determined Disease Status (PDDS) scale, over a 24-month period in a large sample of persons with MS [14]. Interestingly, we observed a progression in disability status over time, but this change was not accompanied by, nor predicted by, a change in self-reported BMI. A comprehensive assessment of mobility may capture different information than that captured by the PDDS alone, and may be affected by weight status in persons with MS, as seen in nondiseased obese populations.

Measures that assess mobility in persons with neurological disorders have been described by Pearson et al. 
[15]. These include timed walking tests, endurance tests, quantitative movement analysis, energy consumption, selfreport questionnaires, and activity monitoring.

We examined the effect of weight status on a comprehensive set of mobility outcomes, including ambulatory performance, $C_{\mathrm{w}}$, spatiotemporal parameters of gait, self-reported walking impairment, and free-living activity in persons with MS. Based on previous research in healthy controls and mobility impairments in persons with MS, we expected that weight status would negatively impact outcomes of mobility, resulting in greater mobility impairment in persons with a higher BMI. Such an examination is important given the prevalence of overweight and obesity in persons with MS and the possible impact this comorbidity may have on health and disease status. If weight status is important to mobility in persons with MS, targeted weight loss interventions may represent alterative and potential adjunct strategies for managing the consequences of this disease.

\section{Methods and Procedures}

2.1. Participants. We recruited a community-residing sample of persons with MS. Participants were included in this investigation based on four criteria: a clinically definite diagnosis of MS; free of a relapse in the past 30 days prior to completing testing procedures; age $\geq 18$ years; and ambulatory either with or without the use of an assistive device.

\subsection{Measures}

2.2.1. Body Mass Index. Height and weight were collected using a calibrated, scale-stadiometer unit (Detecto model 3P7044, Webb City, MO, USA) while participants were wearing light clothing and athletic shoes. BMI was calculated as weight in kilograms divided by height in meters squared. BMI was classified as follows: normal weight, BMI = $18.5-24.9 \mathrm{~kg} / \mathrm{m}^{2}$; overweight, $\mathrm{BMI}=25.0-29.9 \mathrm{~kg} / \mathrm{m}^{2}$; obese, classes I and II, BMI $=30.0-39.9 \mathrm{~kg} / \mathrm{m}^{2}[1]$.

2.2.2. Walking Performance. The timed 25 -foot walk (T25FW) and the 6-minute walk (6MW) assessed walking speed and endurance, respectively. Participants performed two T25FW trials according to standardized instructions [16]. We averaged the time from the two walking trials. The $6 \mathrm{MW}$ was performed in a single corridor that was $75 \mathrm{ft}$ in length or in a square hallway with four corridors that were $100 \mathrm{ft}$ in length, depending on the testing location. Participants were instructed to walk as fast and far as possible for the duration of 6 minutes according to standardized instructions and were permitted to use an assistive device if necessary [13]. Total distance walked in meters was quantified by a member of the research team who followed 1 meter behind the participant with a measuring wheel (Stanley MS50, New Briton, CT, USA).

2.2.3. Oxygen Cost of Walking $\left(C_{w}\right)$. Oxygen consumption $\left(\dot{V}_{2}\right)$ was assessed during the $6 \mathrm{MW}$ by breath-by-breath analysis using a commercially available portable metabolic unit (K4b2, Cosmed, Italy). The $\mathrm{O}_{2}$ and $\mathrm{CO}_{2}$ sensors of the portable metabolic system and the flow meter were calibrated prior to all testing sessions. $\dot{V} \mathrm{O}_{2}(\mathrm{~mL} / \mathrm{kg} / \mathrm{min})$ values collected over the second 3 minutes of the $6 \mathrm{MW}$ were used to generate steady-state $\dot{V} \mathrm{O}_{2}[17] . C_{\mathrm{w}}(\mathrm{mL} / \mathrm{kg} / \mathrm{m})$ was determined by dividing steady state $\dot{V} \mathrm{O}_{2}(\mathrm{~mL} / \mathrm{kg} / \mathrm{min})$ by walking speed $(\mathrm{m} / \mathrm{min})$.

2.2.4. Spatial and Temporal Parameters of Gait. Participants performed two walking trials at a comfortable pace using a GAITRite (CIR Systems, Inc.) electronic walkway. Gait parameters collected included walking velocity $(\mathrm{cm} / \mathrm{s})$, cadence (steps/min), step length $(\mathrm{cm})$, step time (s), double support (percentage of gait cycle), single support (percentage of gait cycle), and base of support $(\mathrm{cm})$. The mean value of the two trials was generated for each of the 7 gait parameters.

2.2.5. Self-Reported Walking Impairment. The MSWS-12 is a 12-item self-report scale that assesses the impact of MS on walking ability [18]. Each of the 12 items is rated on a scale ranging from 1 (not at all) to 5 (extremely). Total scores on the MSWS-12 range from 0-100, whereby higher scores indicate greater walking impairment.

2.2.6. Patient-Determined Disease Steps. The PDDS scale [19, 20 ] was used to determine the level of neurological disability of the participants. The PDDS is a patient-reported scale ranging from 0 (normal) to 8 (bedridden), whereby higher scores indicate greater perceived disability. PDDS scores have correlated strongly $(r=.93)$ with scores on the physicianadministered Expanded Disability Status Scale (EDSS) [21]. Previous data collected by our research group supports a strong $(\rho=.78)$ association between PDDS and EDSS scores.

2.2.7. Free-Living Activity. Daily step and movement counts were assessed over a 7-day period using an ActiGraph accelerometer (model GT3x) (ActiGraph Corporation, Pensacola, FL, USA) worn on a belt around the waist. The accelerometer and this location on the body assess vertical bodily movement using a piezoelectric bender element that produces an electric signal proportional to the force acting upon it. This electric signal is converted into numerical counts generated over preprogrammed 1-minute intervals. Data recorded and stored in the accelerometer were downloaded using ActiLife software and processed using Microsoft Excel. Activity logs completed by participants were used for data verification. Step counts and movement counts for each minute were summed for each of the 7 days and then averaged across the 7-day period.

2.3. Procedures. This study received Institutional Review Board approval. Participants competed testing at one of two locations: the Exercise Neuroscience Laboratory, University of Illinois, Urbana, IL, USA or the Illinois Neurologic Institute, Peoria, IL, USA. Participants provided written informed consent prior to participation in the scheduled 
testing session. Height and weight were collected by a member of the research team. Participants completed ambulatory performance tests including the T25FW and the 6MW. Participants wore a metabolic system during the $6 \mathrm{MW}$ test to assess $C_{\mathrm{w}}$. Spatial and temporal parameters of gait were collected over two walking trials on an electronic walkway. Participants then completed questionnaires assessing the impact of MS on walking impairment and self-reported neurological disability. Upon completion of the testing session, participants were given an accelerometer to wear around the waist over the nondominant hip during the waking hours of the day, except while showering, bathing, and swimming, over 7 consecutive days. Waking hours were defined as the moment of getting out of bed in the morning through the moment of getting into bed at night. Participants were instructed to maintain typical levels of ambulatory activity during this one-week period. Participants were provided with a log sheet to record times when the accelerometer was worn each day. The accelerometer was returned through the US Postal Service in a preaddressed, prestamped envelope provided by the investigators.

2.4. Data Analysis. Descriptive statistics were used to summarize demographic and clinical characteristics of the sample. Values in the text are presented as mean (SD), unless otherwise specified. One-way ANCOVA controlling for age, sex, and disease duration was conducted to determine differences between groups based on BMI classification (i.e., normal weight, overweight, and obese) on T25FW and 6MW performance, $C_{\mathrm{w}}, 7$ spatiotemporal gait parameters, MSWS12 scores, and daily step and movement counts. The 13 measures included herein have been described by Pearson et al. [15] as outcomes for assessing walking mobility in neurological populations. An adjusted alpha level of $P \leq .004$ was therefore used to account for the interrelated outcomes so as to avoid type I errors. Effect sizes (ESs) were calculated to determine the magnitude of the difference in mobility performance between BMI groups expressed as Cohen's $d$ [22]. Guidelines of .2, .5, and .8 were used to determine small, moderate, and large effect sizes, respectively.

\section{Results}

3.1. Participants. Clinical and demographic characteristics of participants are presented in Table 1. This study included 168 persons ( 130 women, 38 men) with predominately a relapsing-remitting disease course (134 relapsing remitting MS; 19 secondary progressive MS; 11 primary progressive MS; 4 not reported). Mean age of participants was 50.9 (10.6) years (range $=27-78$ years) with a mean disease duration of 11.9 (9.3) years (range $=1-43$ years). The median (IQR) PDDS score was 3.0 (3.0) (i.e., gait disability) and ranged from 0 (i.e., normal) through 6 (i.e., bilateral support). Mean BMI was $27.7(5.1) \mathrm{kg} / \mathrm{m}^{2}$ (range $\left.=18.6-39.3 \mathrm{~kg} / \mathrm{m}^{2}\right)$ consistent with previous research involving BMI in MS [4, 5, 23].

3.2. BMI Distribution. The distribution of BMI scores was as follows: $52(31.0 \%)$ participants were classified as normal weight; $61(36.3 \%)$ participants were classified as overweight; and $55(32.7 \%)$ participants were classified as obese. BMI distribution in our study was similar to previous MS samples, although the proportion of participants classified as overweight or obese was somewhat higher in the current investigation [3-5].

3.3. BMI and Outcomes of Mobility. Descriptive characteristics of mobility outcomes and differences along with ESs by BMI classification are presented in Table 2.

3.4. Walking Performance. Mean time to perform the T25FW was 6.7 (4.1) seconds and did not differ significantly by BMI classification, $F(2,160)=0.93, P=.40$. Participants in the sample walked a mean of 420.0 (138.5) meters during the $6 \mathrm{MW}$ test which was not significantly different based on BMI classification, $F(2,161)=3.11, P=.05$. Small ESs were observed for the difference between normal and obese groups on T25FW performance (.29), and between normal and overweight groups on 6MW performance (-.27).

3.5. $C_{w}$. Mean $C_{\mathrm{w}}$ in the sample was $0.225(0.119) \mathrm{mL} / \mathrm{kg} / \mathrm{m}$. There was no significant difference between groups with respect to $C_{\mathrm{w}}, F(2,157)=3.32, P=0.04$, although a moderate ES (.62) was observed for the difference in $C_{\mathrm{w}}$ between normal weight and obese groups. The magnitude of the difference in $C_{\mathrm{w}}$ between normal and overweight groups $(-.27)$, and between overweight and obese groups (.20) was small.

3.6. Spatial and Temporal Parameters of Gait. None of the spatiotemporal gait parameters examined differed based on BMI: walking velocity, $F(2,159)=2.53, P=0.08$; cadence, $F(2,159)=1.64, P=0.20$; step length, $F(2,159)=1.70$, $P=0.19$; step time, $F(2,159)=1.00, P=0.37$; percentage of gait cycle spent in double support, $F(2,159)=.50, P=0.61$; percentage of gait cycle spent in single support, $F(2,159)=$ $.49, P=0.61$; base of support, $F(2,159)=0.37, P=0.69$. The magnitude of the difference between groups on gait parameters ranged from no effect to small ( range $=-.02$ to $.34)$.

3.7. Self-Reported Walking Impairment. Mean MSWS-12 score for the sample was 42.8 (28.5) and was not significantly different based on BMI classification, $F(2,162)=1.38, P=$ .26 .

3.8. Free Living Mobility. Mean daily step and movement counts were 4215.5 (2469.6) and 144725.7 (84828.8), respectively. There was no difference between BMI groups in free living mobility assessed by daily step, $F(2,159)=2.43, P=$ 0.09 , and daily movement counts, $F(2,159)=1.15, P=0.32$. The magnitude of the difference in daily step counts between groups was small when normal weight and overweight groups were compared $(-.28)$, and when overweight and obese groups were compared (.31). 
TABLE 1: Descriptive and clinical characteristics of the total MS sample and by BMI classification.

\begin{tabular}{|c|c|c|c|c|}
\hline Characteristics & $\begin{array}{l}\text { Normal weight } \\
\qquad(N=52)\end{array}$ & $\begin{array}{c}\text { Overweight } \\
(N=61)\end{array}$ & $\begin{array}{c}\text { Obese } \\
(N=55)\end{array}$ & $\begin{array}{c}\text { Total } \\
(N=168)\end{array}$ \\
\hline Age, years & $48.5(11.6)$ & $51.5(9.5)$ & $52.5(10.6)$ & $50.9(10.6)$ \\
\hline Sex, female/male & $42 / 10$ & $47 / 14$ & $42 / 14$ & $130 / 38$ \\
\hline BMI, $\mathrm{kg} / \mathrm{m}^{2}$ & $22.0(1.7)$ & $27.2(1.5)$ & $33.7(2.7)$ & $27.7(5.1)$ \\
\hline Weight, kg & $62.7(8.2)$ & $76.6(8.0)$ & $97.6(12.9)$ & $79.1(17.2)$ \\
\hline Height, $\mathrm{cm}$ & $168.6(8.2)$ & $167.2(9.3)$ & $170.0(9.8)$ & $168.6(9.1)$ \\
\hline PDDS & $3.0(3.0)$ & $3.0(3.0)$ & $3.0(3.0)$ & $3.0(3.0)$ \\
\hline Disease duration, years & $13.8(9.6)$ & $10.9(7.8)$ & $11.2(10.3)$ & $11.9(9.3)$ \\
\hline $\begin{array}{l}\text { Disease course, } \\
\text { RRMS/SPMS/PPMS/NR }\end{array}$ & $43 / 8 / 0 / 1$ & $45 / 7 / 7 / 2$ & $46 / 4 / 4 / 1$ & $134 / 19 / 11 / 4$ \\
\hline
\end{tabular}

Values are mean (SD), PDDS is median (IQR). BMI: body mass index; PDDS: patient-determined disease steps; RRMS: relapsing-remitting MS; SPMS: secondary progressive MS; PPMS: primary progressive MS; NR: not reported.

TABLE 2: Descriptive characteristics and effect sizes for mobility outcomes by BMI classification.

\begin{tabular}{|c|c|c|c|c|c|c|}
\hline \multirow[b]{2}{*}{ Outcome } & \multicolumn{3}{|c|}{ Descriptive characteristics } & \multicolumn{3}{|c|}{ Effect sizes } \\
\hline & Normal weight & Overweight & Obese & $\begin{array}{c}\text { Normal } \\
\text { versus } \\
\text { Overweight }\end{array}$ & $\begin{array}{c}\text { Normal } \\
\text { versus } \\
\text { Obese }\end{array}$ & $\begin{array}{c}\text { Overweight } \\
\text { versus } \\
\text { Obese }\end{array}$ \\
\hline \multicolumn{7}{|l|}{ Walking performance } \\
\hline T25FW, s & $7.1(4.3)$ & $6.9(5.1)$ & $6.2(2.2)$ & .05 & .29 & .18 \\
\hline $6 \mathrm{MW}, \mathrm{m}$ & $396.4(175.4)$ & $438.4(131.7)$ & $422.2(100.3)$ & -.27 & -.19 & .14 \\
\hline \multicolumn{7}{|l|}{ Walking cost } \\
\hline$C_{\mathrm{w}}, \mathrm{mL} / \mathrm{kg} / \mathrm{m}$ & $0.256(0.136)$ & $0.221(0.144)$ & $0.202(0.046)$ & .25 & .62 & .20 \\
\hline \multicolumn{7}{|l|}{ Spatiotemporal gait } \\
\hline Velocity, $\mathrm{cm} / \mathrm{s}$ & $97.1(30.8)$ & $105.6(28.9)$ & $107.1(29.9)$ & -.28 & -.33 & -.05 \\
\hline Cadence, steps/min & $100.6(14.9)$ & $103.6(17.8)$ & $105.5(14.7)$ & -.19 & -.33 & -.12 \\
\hline Step length, $\mathrm{cm}$ & $56.5(13.2)$ & $60.0(11.5)$ & $59.5(12.0)$ & -.28 & -.24 & .04 \\
\hline Step time, s & $0.624(0.116)$ & $0.618(0.196)$ & $0.588(0.095)$ & .04 & .34 & .21 \\
\hline Double support, gait cycle (\%) & $32.5(6.2)$ & $31.6(6.9)$ & $32.8(5.7)$ & .13 & -.05 & -.18 \\
\hline Single support, gait cycle (\%) & $34.2(3.6)$ & $34.3(3.4)$ & $33.7(2.9)$ & -.03 & .17 & .21 \\
\hline Base of support, $\mathrm{cm}$ & $12.3(5.1)$ & $11.7(3.6)$ & $12.4(3.6)$ & .13 & -.02 & -.18 \\
\hline \multicolumn{7}{|l|}{ Self-reported walking } \\
\hline MSWS-12 & $45.8(32.0)$ & $40.5(27.8)$ & $42.3(26.3)$ & .18 & .12 & -.07 \\
\hline \multicolumn{7}{|l|}{ Free-living activity } \\
\hline Daily step count & $3951.0(2404.1)$ & $4701.1(2913.8)$ & $3953.6(1926.6)$ & -.28 & -.001 & .31 \\
\hline Daily movement count & $139562.7(88691.9)$ & $154247.3(95943.1)$ & $139566.3(67516.0)$ & -.16 & $<.001$ & .18 \\
\hline
\end{tabular}

Values are mean (SD). BMI: body mass index; T25FW: timed 25-foot walk; 6MW: 6-minute walk; $C_{\mathrm{w}}$ : oxygen cost of walking; MSWS-12: Multiple Sclerosis Walking Scale- 12.

\section{Discussion}

This is the first study to provide descriptive and comparative data regarding objectively measured weight status and its association with a comprehensive set of mobility outcomes in a large sample of ambulatory persons with MS. Almost 70\% of the sample were identified as overweight or obese, classes I and II (i.e., BMI = 25.0-39.9); however, weight status did not appear to have a consistent, large, or significant impact on mobility outcomes. Nevertheless, the high prevalence of overweight and obesity in this sample may still have important health or disease consequences for persons with MS, but these seemingly do not involve mobility dysfunction. Further, this emphasizes the importance of identifying and focusing on other factors which may be important to mobility in persons with MS.

We did not establish any significant difference between BMI status on T25FW and 6MW performance, $C_{\mathrm{w}}$, spatiotemporal gait parameters, MSWS-12 scores, and freeliving mobility outcomes. This was supported by generally 
small ESs when comparing the magnitude of the difference between groups on mobility outcomes. Overall, these findings are contrary to our expectations based on the influence of excess body weight on mobility outcomes established in nondiseased adult populations [7-9]. That body of research suggests superior ambulatory performance in normal-weight individuals without a chronic disease or condition. Factors other than weight status might account for ambulatory problems in persons with MS and such factors are different from adults without a disease. Factors which may contribute to impaired walking in persons with MS as outlined by Pearson et al. [15] include muscle weakness, spasticity, loss of proprioception and coordination, vestibular and visual dysfunction, cognitive and mood disturbances, and pain.

To further evaluate and confirm the relationship between weight status and mobility, we conducted partial correlations between BMI and outcomes of mobility controlling for age, sex, and disease duration (data not presented). There was a mean partial correlation coefficient (SD) of .01 (.12) which ranged from -.21 to .16. This further supports the evidence presented herein indicating weak, inconsistent associations between BMI and mobility in persons with MS; such associations indicate that, at best, less than $4 \%$ of variance is shared between BMI and mobility outcomes. Our findings are further in accordance with cross-sectional $[5,24]$ and prospective [14] data which do not support a relationship between weight status (determined by BMI and percent body fat) and disability (determined by the EDSS, PDSS, and a self-report functional ability) in persons with MS. The present study further provides a comprehensive assessment of the functional impact of weight status on both clinical and community mobility outcomes in a large sample of persons with MS which may provide different information than that captured by disability measures alone. Collectively, this suggests that the effect of weight status on mobility is limited in persons with MS and that this relationship may differ from that established in the general nondiseased population. The lack of an association between weight status and mobility is important in suggesting that we should focus on other factors that may impact mobility in persons with MS.

Interestingly, a potential role for weight status in the development of MS has been suggested. Before the age of 20, a high BMI (>27.0-30.0) has been associated with a greater than twofold increase in the risk of developing MS $[25,26]$. No association, however, was established between adult BMI and risk of MS [25, 26]. Taken together with our current and previous findings [14], this suggests that weight status may be more influential during disease development than following disease onset, although this concept requires further investigation.

The combined prevalence of overweight and obesity, classes I and II (i.e., BMI = 25.0-39.9) in the current sample was 69.0 percent. This percentage is somewhat higher than values that have been observed by other investigators but is comparable to the general nondiseased adult population. For instance, 55.6 percent of individuals were classified as overweight and obese in a large sample of persons with MS $(n=8983)$ [4]. In a sample of veterans with MS,
53.0 percent of women and 63.3 percent of men were classified as overweight and obese [3]. Similarly, 53.2 percent of persons with MS were classified as overweight or obese in a previous investigation by our research group [14]. Those studies all used self-reported height and weight which may have led to an underestimation of overweight and obesity in these investigations. Furthermore, our findings are consistent with those of the general nondiseased adult population in which 68.0 percent of persons were identified as overweight and obese [6]. Height and weight were assessed by the investigators using a scale stadiometer, rather than self-reported, in that cross-sectional, nationwide sample of healthy adults without disease [6]. Our results suggest BMI may be somewhat higher than previously thought in persons with MS, but similar to estimates in nondiseased adult populations.

We observed that weight status does not appear to impact mobility outcomes, but the prevalence of excess body mass may still pose considerable health risks for persons with MS. Obesity is a risk factor for a variety of chronic health conditions including diabetes, hypertension, high cholesterol, stroke, heart disease, certain cancers, and arthritis [27]. Obesity further is associated with cognitive outcomes in the general population [26] and this might account, in part, for the extensive prevalence and burden of cognitive impairment in MS. Persons with MS and health care providers should be aware and address additional health risks imposed by excess body weight.

The present study provides comparative information on weight status and its effect on a comprehensive battery of mobility outcomes in a large sample of persons with MS. Nevertheless, the generalizability of our conclusions is mainly limited to ambulatory, community-residing persons with MS who have a relapsing-remitting disease course. This research used BMI as a marker of weight status in persons with MS. Other outcomes such as waist circumference, body shape and composition, or fat distribution may have had different associations with mobility outcomes than those observed using BMI alone. Future studies should include gold standard outcomes for the assessment of body composition, such as dual-energy X-ray absorptiometry (DXA), to further establish the relationship between weight status and mobility in persons with MS. This study might benefit from the inclusion of longitudinal data to determine the possible impact of changes in weight status on mobility outcomes over time, although our previous data using selfreport measures would argue against such a prospective association between variables [14]. The inclusion of symptomatic outcomes known to be influenced by obesity, such as pain and fatigue, may further impact mobility and should be included in future investigations.

\section{Conclusion}

We provide the first evaluation of the effect of BMI on a comprehensive battery of mobility outcomes including ambulatory performance, $C_{\mathrm{w}}$, spatiotemporal parameters of gait, self-reported walking impairment, and free-living 
activity. There does not appear to be a consistent nor strong pattern of association between weight status and mobility, which differs from results observed in nondiseased adult populations. This highlights the importance of focusing on other factors that may impact mobility in persons with MS. The high proportion of persons with MS classified as overweight or obese in the current sample, nevertheless, remains a considerable health concern that requires better management.

\section{Conflict of Interests}

The authors declare that they have no conflict of interests.

\section{Acknowledgments}

Support for this study was provided by a grant from the OSF Hospital and National Multiple Sclerosis Society (PP 1695). L. A. Pilutti was the recipient of a Du Pré Grant from the Multiple Sclerosis International Federation.

\section{References}

[1] K. A. Donato, "Executive summary of the clinical guidelines on the identification, evaluation, and treatment of overweight and obesity in adults," Archives of Internal Medicine, vol. 158, no. 17, pp. 1855-1867, 1998.

[2] WHO, Expert Committee on Physical Status. Physical Status: The Use and Interpretation of Anthropometry, World Health Organization, Geneva, Switzerland, 1995.

[3] S. R. Khurana, A. M. Bamer, A. P. Turner et al., "The prevalence of overweight and obesity in veterans with multiple sclerosis," American Journal of Physical Medicine and Rehabilitation, vol. 88, no. 2, pp. 83-91, 2009.

[4] R. A. Marrie, R. Horwitz, G. Cutter, T. Tyry, D. Campagnolo, and T. Vollmer, "High frequency of adverse health behaviors in multiple sclerosis," Multiple Sclerosis, vol. 15, no. 1, pp. 105113, 2009.

[5] J. N. Slawta, A. R. Wilcox, J. A. McCubbin, D. J. Nalle, S. D. Fox, and G. Anderson, "Health behaviors, body composition, and coronary heart disease risk in women with multiple sclerosis," Archives of Physical Medicine and Rehabilitation, vol. 84, no. 12, pp. 1823-1830, 2003.

[6] K. M. Flegal, M. D. Carroll, B. K. Kit, and C. L. Ogden, "Prevalence of obesity and trends in the distribution of body mass index among US adults, 1999-2010," Journal of the American Medical Association, vol. 307, no. 5, pp. 491-497, 2012.

[7] M. Hulens, G. Vansant, A. L. Claessens, R. Lysens, and E. Muls, "Predictors of 6-minute walk test results in lean, obese and morbidly obese women," Scandinavian Journal of Medicine and Science in Sports, vol. 13, no. 2, pp. 98-105, 2003.

[8] R. C. Browning, E. A. Baker, J. A. Herron, and R. Kram, "Effects of obesity and sex on the energetic cost and preferred speed of walking," Journal of Applied Physiology, vol. 100, no. 2, pp. 390-398, 2006.

[9] P. P. K. Lai, A. K. L. Leung, A. N. M. Li, and M. Zhang, "Three-dimensional gait analysis of obese adults," Clinical Biomechanics, vol. 23, no. 1, pp. S2-S6, 2008.

[10] U. Givon, G. Zeilig, and A. Achiron, "Gait analysis in multiple sclerosis: characterization of temporal-spatial parameters using GAITRite functional ambulation system," Gait and Posture, vol. 29, no. 1, pp. 138-142, 2009.

[11] J. J. Sosnoff, B. M. Sandroff, and R. W. Motl, "Quantifying gait abnormalities in persons with multiple sclerosis with minimal disability," Gait and Posture, vol. 36, no. 1, pp. 154-156, 2012.

[12] R. W. Motl, Y. Suh, D. Dlugonski et al., "Oxygen cost of treadmill and over-ground walking in mildly disabled persons with multiple sclerosis," Neurological Sciences, vol. 32, no. 2, pp. 255-262, 2011.

[13] M. D. Goldman, R. A. Marrie, and J. A. Cohen, "Evaluation of the six-minute walk in multiple sclerosis subjects and healthy controls," Multiple Sclerosis, vol. 14, no. 3, pp. 383-390, 2008.

[14] L. A. Pilutti, E. McAuley, and R. W. Motl, "Weight status and disability in multiple sclerosis: an examination of bidirectional associations over a 24-month period," Multiple Sclerosis and Related Disorders, vol. 1, no. 3, pp. 139-144, 2012.

[15] O. R. Pearson, M. E. Busse, R. W. M. Van Deursen, and C. M. Wiles, "Quantification of walking mobility in neurological disorders," Monthly Journal of the Association of Physicians, vol. 97, no. 8, pp. 463-475, 2004.

[16] J. S. Fischer, A. J. Jak, J. E. Knicker, R. A. Rudick, and G. I. Cutter, Administration and Scoring Manual for the Multiple Sclerosis Functional Composite Measure (MSFC), Demos Medical, New York, NY, USA, 1999.

[17] R. W. Motl, B. M. Sandroff, Y. Suh, and J. J. Sosnoff, "Energy cost of walking and its association with gait parameters, daily activity, and fatigue in persons with mild multiple sclerosis," Neurorehabilitation and Neural Repair, vol. 26, no. 8, pp. 10511021, 2012.

[18] J. C. Hobart, A. Riazi, D. L. Lamping, R. Fitzpatrick, and A. J. Thompson, "Measuring the impact of MS on walking ability: the 12-item MS Walking Scale (MSWS-12)," Neurology, vol. 60 , no. 1, pp. 31-36, 2003.

[19] M. J. Hohol, E. J. Orav, and H. L. Weiner, "Disease steps in multiple sclerosis: a simple approach to evaluate disease progression," Neurology, vol. 45, no. 2, pp. 251-255, 1995.

[20] M. J. Hohol, E. J. Orav, and H. L. Weiner, "Disease steps in multiple sclerosis: a longitudinal study comparing Disease Steps and EDSS to evaluate disease progression," Multiple Sclerosis, vol. 5, no. 5, pp. 349-354, 1999.

[21] O. Hadjimichael, R. D. Kerns, M. A. Rizzo, G. Cutter, and T. Vollmer, "Persistent pain and uncomfortable sensations in persons with multiple sclerosis," Pain, vol. 127, no. 1-2, pp. 35-41, 2007.

[22] J. Cohen, Statistical Power Analysis for the Behavioral Sciences, Lawrence Erlbaum Associates, Hillsdale, NJ, USA, 2nd edition, 1988.

[23] M. C. Mojtahedi, E. M. Snook, R. W. Motl, and E. M. Evans, "Bone health in ambulatory individuals with multiple sclerosis: impact of physical activity, glucocorticoid use, and body composition," Journal of Rehabilitation Research and Development, vol. 45, no. 6, pp. 851-861, 2008.

[24] C. P. Lambert, R. Lee Archer, and W. J. Evans, "Body composition in ambulatory women with multiple sclerosis," Archives of Physical Medicine and Rehabilitation, vol. 83, no. 11, pp. 1559-1561, 2002.

[25] A. K. Hedström, T. Olsson, and L. Alfredsson, "High body mass index before age 20 is associated with increased risk for multiple sclerosis in both men and women," Multiple Sclerosis, vol. 18, no. 9, pp. 1334-1336, 2012.

[26] E. Smith, P. Hay, L. Campbell, and J. N. Trollor, "A review of the association between obesity and cognitive function across the lifespan: implications for novel approaches to prevention 
and treatment," Obesity Reviews, vol. 12, no. 9, pp. 740-755, 2011.

[27] S. D. H. Malnick and H. Knobler, "The medical complications of obesity," Quarterly Journal of Medicine, vol. 99, no. 9, pp. 565-579, 2006. 


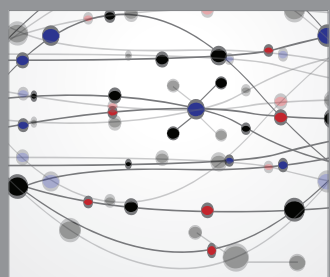

The Scientific World Journal
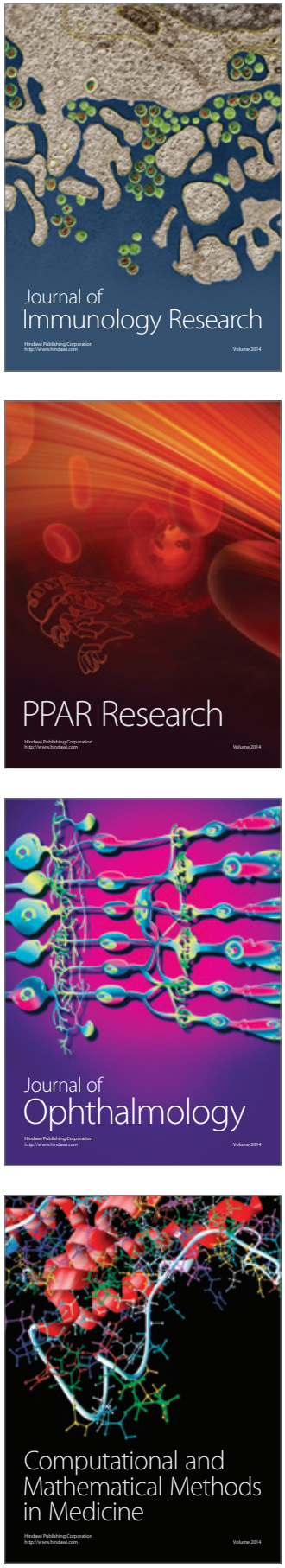

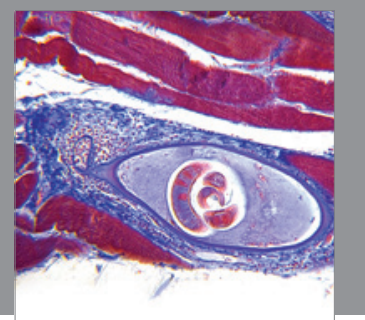

Gastroenterology

Research and Practice
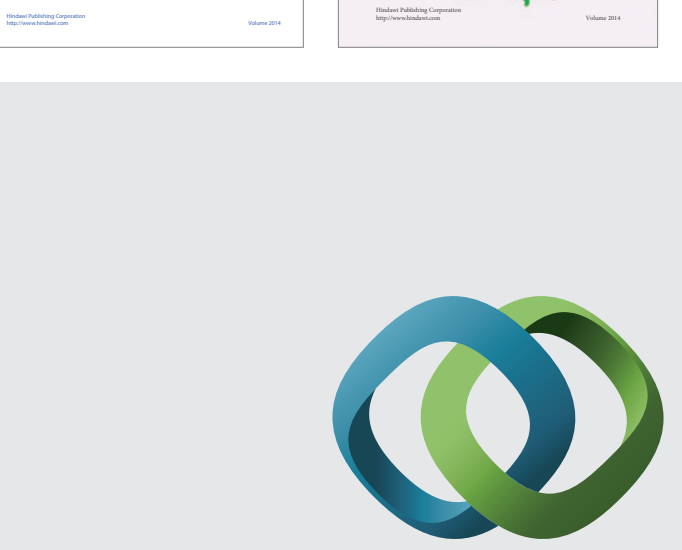

\section{Hindawi}

Submit your manuscripts at

http://www.hindawi.com
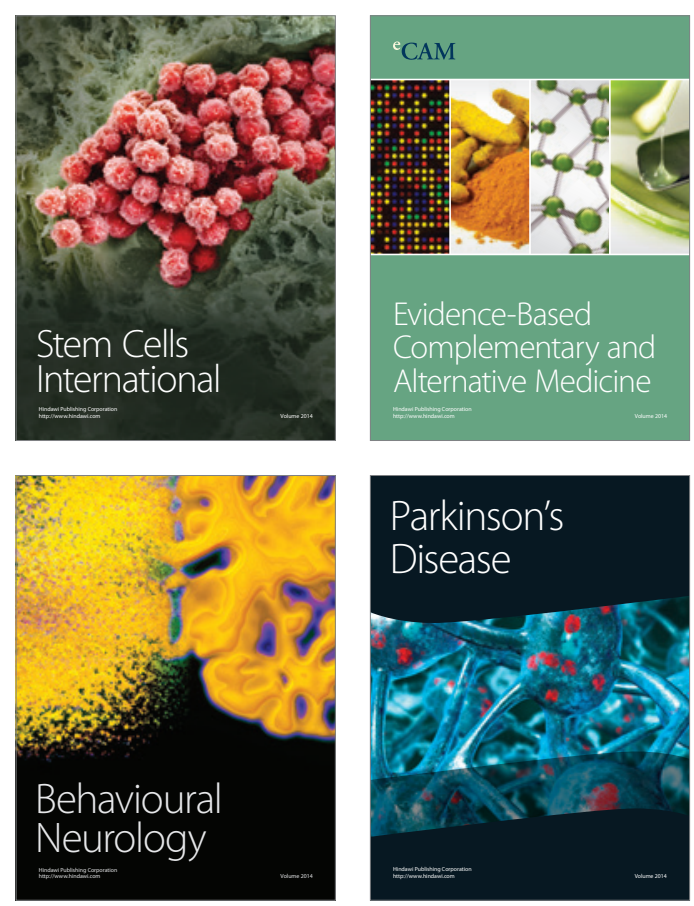

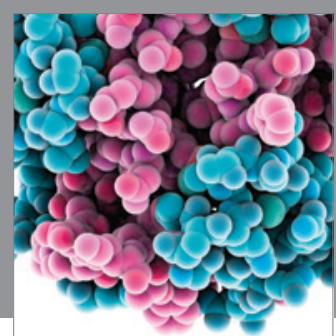

Journal of
Diabetes Research

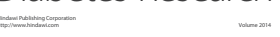

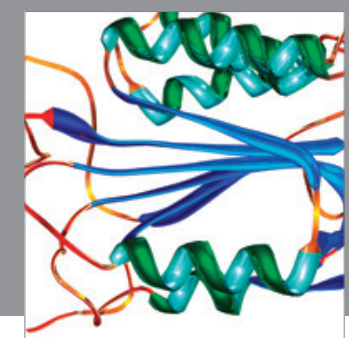

Disease Markers
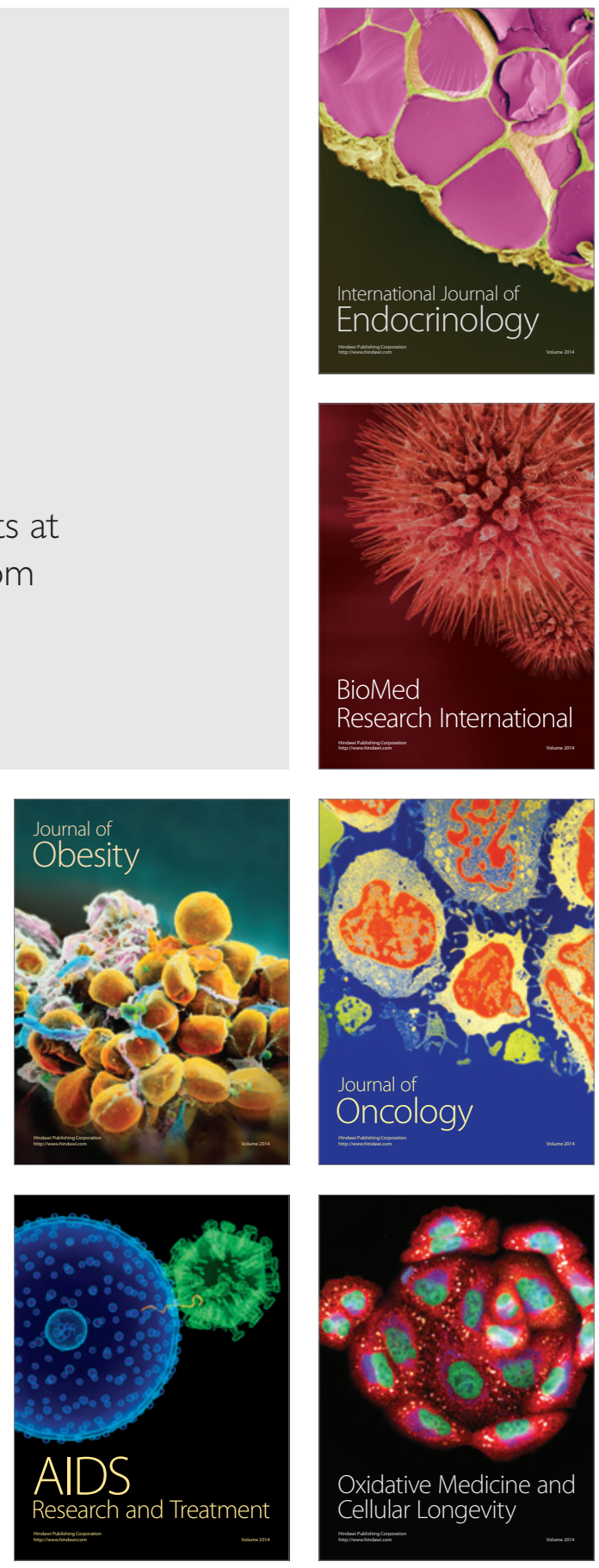